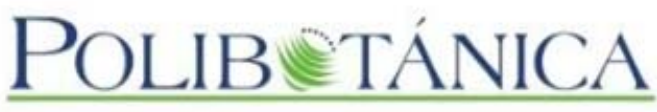

\title{
Polibotánica
}

ISSN electrónico: 2395-9525

polibotanica@gmail.com

Instituto Politécnico Nacional

México

http://www.polibotanica.mx

\section{VARIACIÓN ESTACIONAL DE LAS ASCOSPORAS DE Helicogermslita (XYLARIACEAE) EN LA ATMÓSFERA DE BAHÍA BLANCA, ARGENTINA.}

\section{SEASONAL VARIATION OF Helicogermslita (XYLARIACEAE) ASCOSPORES IN THE ATMOSPHERE OF BAHÍA BLANCA CITY, ARGENTINA.}

Castillo, L.A., M. V. Bianchinotti y M. G. Murray.

VARIACIÓN ESTACIONAL DE LAS ASCOSPORAS DE Helicogermslita (XYLARIACEAE) EN LA ATMÓSFERA DE BAHÍA BLANCA, ARGENTINA. SEASONAL VARIATION OF Helicogermslita (XYLARIACEAE) ASCOSPORES IN THE ATMOSPHERE OF BAHÍA BLANCA CITY, ARGENTINA.

POLIBETANICA

Instituto Politécnico Nacional
Núm. 49: 173-184 México. Enero 2020

DOI: $10.18387 /$ polibotanica.49.12 


\section{VARIACIÓN ESTACIONAL DE LAS ASCOSPORAS DE Helicogermslita (XYLARIACEAE) EN LA ATMÓSFERA DE BAHÍA BLANCA, ARGENTINA.}

\section{SEASONAL VARIATION OF Helicogermslita (XYLARIACEAE) ASCOSPORES IN THE ATMOSPHERE OF BAHÍA BLANCA CITY, ARGENTINA.}

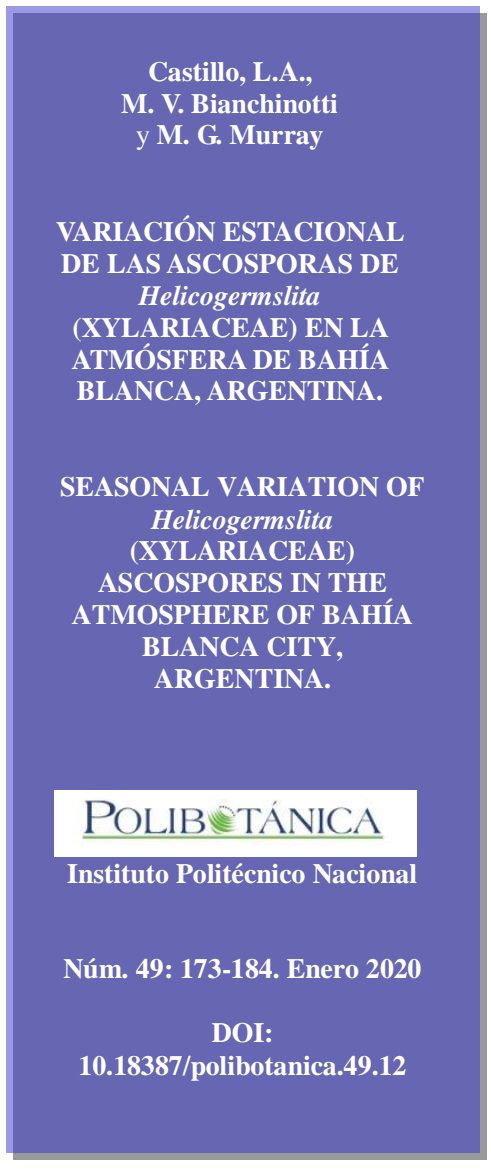

\author{
L. A. Castillo \\ Instituto de Ciencias Biológicas y Biomédicas del Sur INBIOSUR \\ (CONICET - UNS). Departamento de Biología, Bioquímica y Farmacia \\ Universidad Nacional del Sur.
}

M. V. Bianchinotti

Centro de Recursos Naturales Renovables de la Zona Semiárida CERZOS (CONICET - UNS). Departamento de Biología, Bioquímica y Farmacia Universidad Nacional del Sur.

M. G. Murray / mgmurray@ criba.edu.ar Instituto de Ciencias Biológicas y Biomédicas del Sur INBIOSUR (CONICET - UNS). Departamento de Biología, Bioquímica y Farmacia

Universidad Nacional del Sur.

RESUMEN: Ascosporas asignables a Helicogermslita (Xylariaceae, Ascomycota) fueron registradas en muestras de aire de la ciudad de Bahía Blanca. Las xilariáceas tienen diversos roles ecológicos (como fitopatógenos, endófitos, descomponedores de madera, productores de múltiples metabolitos secundarios, etc.). El objetivo del trabajo fue determinar a qué especie de Helicogermslita correspondía el tipo esporal hallado, y analizar su dinámica de dispersión en la atmósfera de la ciudad de Bahía Blanca. Las muestras se tomaron diariamente con un equipo volumétrico tipo Hirst ubicado en una zona residencial a $12 \mathrm{~m}$ de altura. Durante el año 2016, se registraron las concentraciones diarias de 27 diferentes tipos esporales, entre ellos, el tipo Helicogermslita. El índice anual de esporas para el tipo Helicogermslita fue de 145 esporas $/ \mathrm{m}^{3}$ de aire, cifra que representó un $0.02 \%$ en relación al total de esporas fúngicas halladas. La mayor representación ocurrió durante el verano y otoño, mientras que el invierno resultó la estación en donde estuvo menos representada. Al analizar la relación entre la aparición de esporas y algunos factores meteorológicos, la humedad relativa constituyó el factor más importante, registrándose correlación positiva y significativa entre él y la concentración de esporas. Las esporas halladas en el aire de Bahía Blanca fueron identificadas como $H$. cf. celastri. No pudo establecerse el sitio de origen de las esporas.

Palabras clave: aire; esporas fúngicas; Ascomycota; muestreador Hirst; aerobiología.

ABSTRACT: The genus Helicogermslita was registered in several European countries, India, New Zeeland, South Africa and Chile. While the natural habitat of the species are plant substrates, in Argentina ascospores from Helicogermslita were observed in air samples from Bahía Blanca city. Members of Xylariaceae are recognized as plant pathogens, endophytes or wood decomposers. The aim of this work was to identify the spore type named after Helicogermslita and to analyze its dynamics of dispersion in Bahía Blanca atmosphere. Samples were taken weekly with a Lanzoni sampler (Hirst methodology) located in a residential area 15 meters high. Daily and schedules data of the concentration from Helicogermslita spores were analyzed during 
2016. The annual index of Helicogermslita spore type was of $145 \mathrm{spores} / \mathrm{m}^{3}$, that represented $0.02 \%$ in relation to the total fungal spores found. The highest values were observed in summer and fall, while winter turned out to be the least represented season. The statistical analyses showed a relationship between the appearance of spores and some meteorological parameters, the relative humidity was the most important factor responsible of the increase in concentration of spores with a significant positive correlation. These ascospores were identified as $H$. cf. celastri. The site of origin of the spores could not be established.

Key words: air; fungal spores; Ascomycota; Hirst sampler; aerobiology.

\section{INTRODUCCIÓN}

El género Helicogermslita (Xylariaceae, Ascomycota) fue descrito por primera vez por Hawksworth y Lodha (1983). Originalmente monotípico, el género cuenta en la actualidad con nueve especies: $H$. aucklandica (Rabenh.) L.E. Petrini, H. celastri (S.B. Kale \& S.V.S. Kale) Lodha \& D. Hawksw., H. diversa S. J. Lee \& Crous, H. fleischhakii (Auersw.) Læssøe \& Spooner, H. gaudefroyi (Fabre) Læssøe \& Spooner, H. gisbornia L.E. Petrini, H. johnstonii L.E. Petrini, H. mackenziei L.E. Petrini, y H. valdiviensis (Speg.) Laessoe \& Spooner (Mycobank 2019 ${ }^{1}$ ). Sus caracteres diagnósticos son los estromas reducidos con las bases periteciales inmersas en el sustrato, y las ascosporas con un surco germinativo en espiral, que va de un extremo a otro, con dos o más giros visibles en la superficie. Las especies de Helicogermslita han sido descriptas creciendo sobre corteza, madera decorticada o fuertemente descompuesta, y se han registrado en Alemania (Læssøe y Spooner, 1993; Rappaz, 1995), Chile, Francia, India (Hawksworth y Lodha , 1983), Nueva Zelanda (Petrini, 2003) y Sudáfrica (Lee y Crous, 2003).

Ascosporas de Helicogermslita han sido registradas también en muestras de diverso origen. Es escaso lo que se conoce sobre la importancia del género, aun cuando otros integrantes de la familia Xylariaceae poseen un importante rol ecológico como fitopatógenos, endófitos o descomponedores de madera (Petrini et al., 1987; Whalley, 1996). Por ello, se consideró de interés estudiar la representación y dinámica de dispersión de las esporas de Helicogermslita en el aire como primera aproximación al estudio de su biología.

El objetivo del trabajo fue determinar a qué especie de Helicogermslita correspondían las ascosporas registradas, y analizar su dinámica de dispersión en la atmósfera de la ciudad de Bahía Blanca.

\section{MATERIAL Y MÉTODOS}

Área de muestreo

La ciudad de Bahía Blanca se encuentra en el extremo sudoeste de la Provincia de Buenos Aires, en el interior del Estuario de Bahía Blanca ( $38^{\circ} 44^{\prime} \mathrm{S}, 62^{\circ} 16^{\prime} \mathrm{O}$ ), Argentina. Su clima es templado/mesotermal con precipitaciones constantes a lo largo del año y veranos calurosos (Kottek et al., 2006); (fig. 1). Las temperaturas medias mensuales oscilan entre $8^{\circ} \mathrm{C}$ (en el mes de julio) y $23.5^{\circ} \mathrm{C}$ (en el mes de enero). Los datos históricos (valores medios 1981-2010) muestran una precipitación media anual promedio de $651 \mathrm{~mm}$ (Servicio Meteorológico Nacional ${ }^{2}$ ). El clima está influenciado por el Océano Atlántico, el cual actúa como un efecto moderador de la temperatura. Sin embargo, el área se encuentra localizada sobre el borde de un estuario y posee, por tanto, también características de continentalidad.

\footnotetext{
${ }^{1}$ https://http://www.mycobank.org

${ }^{2}$ https://www.smn.gob.ar/estadisticas
} 


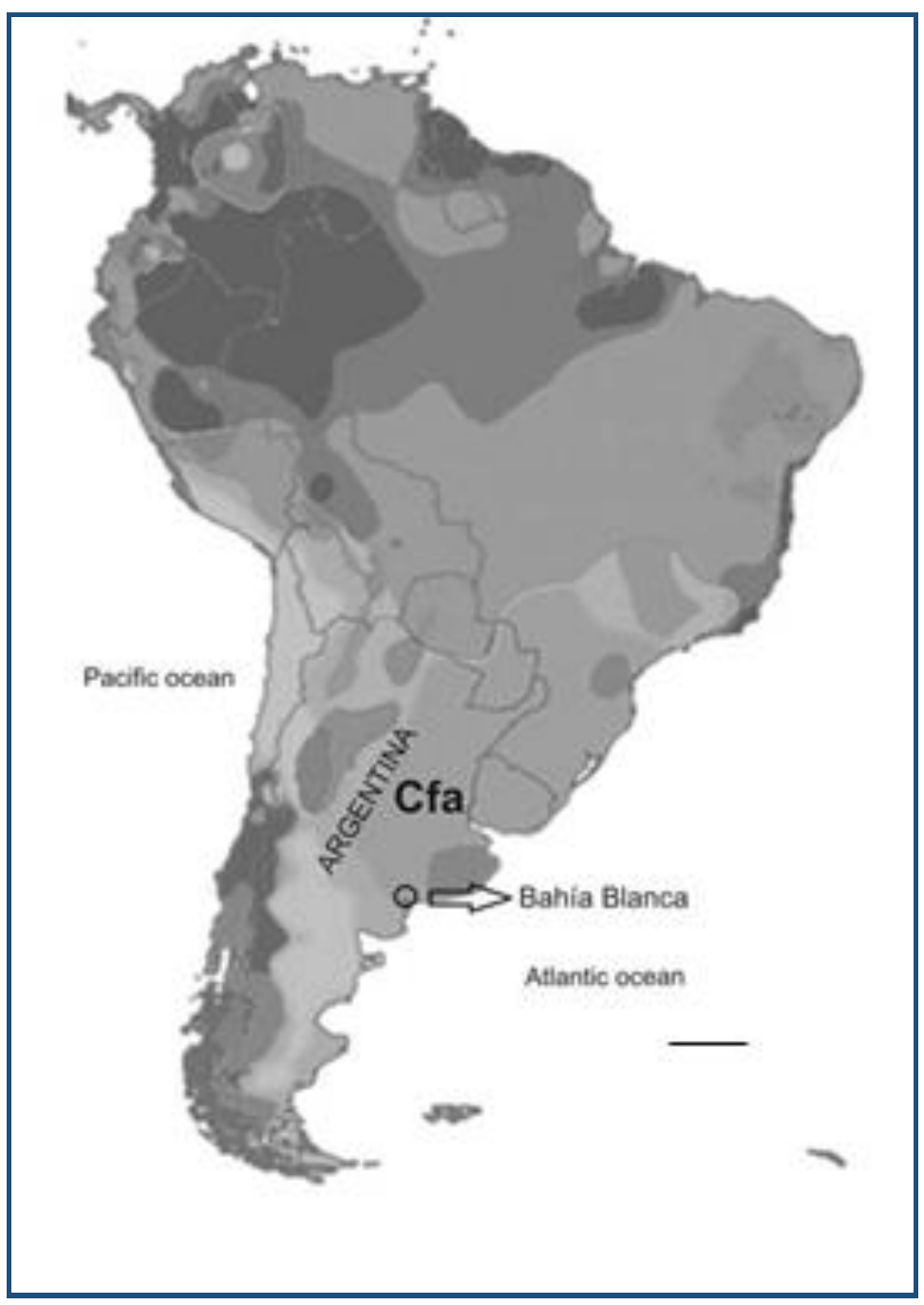

Fig. 1. Ubicación del área de estudio y clasificación climática de Köppen-Geiger. C: templado cálido; f: precipitaciones constantes a lo largo de todo el año; a: verano caluroso.

Figura tomada de Kottek et al., 2006. Barra $=500 \mathrm{~km}$.

Muestreo del aire

El muestreo de las esporas fúngicas aerotransportadas se llevó a cabo desde el 1 de enero hasta el 31 de diciembre de 2016. Se utilizó un equipo volumétrico tipo Hirst (Lanzoni modelo VPPS-2000), localizado a $12 \mathrm{~m}$ de altura, en una terraza con libre circulación de las masas de aire, sin barreras arquitectónicas ni florísticas que entorpecieran el muestreo. Para su instalación, funcionamiento y recuento se siguieron las pautas recomendadas por la REA (Galán et al., 2007). La observación de los preparados se realizó con un microscopio óptico Carl Zeiss (Primo Star). Las esporas fúngicas se identificaron utilizando un atlas de referencia (Grant Smith, 1990). Se registraron las concentraciones diarias de 27 diferentes tipos esporales, entre ellos, el tipo Helicogermslita. Los datos se expresaron como concentración diaria de esporas por metro cúbico de aire (esporas $/ \mathrm{m}^{3}$ ). Para cada ascospora de Helicogermslita se registraron sus dimensiones (longitud y ancho), caracteres del surco y presencia de apéndices. Dichos atributos se compararon con los de las especies aceptadas en el género. 
Datos meteorológicos

Los datos meteorológicos diarios fueron provistos por el Servicio Meteorológico Nacional de Argentina. Las variables meteorológicas consideradas fueron: temperatura media, máxima y mínima $\left({ }^{\circ} \mathrm{C}\right)$, humedad relativa $(\%)$, precipitación del día de estudio y del día anterior $(\mathrm{mm})$ y velocidad del viento $(\mathrm{km} / \mathrm{h})$.

\section{Análisis de datos}

Se calculó el índice anual de esporas (InAEs), definido como la sumatoria de las concentraciones diarias promedio durante el periodo de tiempo estudiado (Galán et al., 2017). El cálculo de este índice permite dimensionar la abundancia de la espora a lo largo del año.

Los análisis estadísticos comprendieron test de normalidad de los datos (prueba Shapiro-Wilk) y correlación no paramétrica de Spearman, entre los factores meteorológicos y el contenido diario de esporas $/ \mathrm{m}^{3}$. Los análisis estadísticos se realizaron con el programa Statistica 10.

\section{RESUlTADOS}

Con el objetivo de establecer la identidad específica de las esporas registradas como tipo esporal Helicogermslita, se compararon los atributos morfológicos de importancia diagnóstica (tamaño, forma, cantidad de giros del surco y presencia de apéndices) con los descriptos en las especies actualmente aceptadas en el género. Dicha comparación y las referencias bibliográficas que la respaldan se presentan en la tabla 1. En la figura 2 se ilustra de manera comparada la morfología de las ascosporas de Helicogermslita spp.

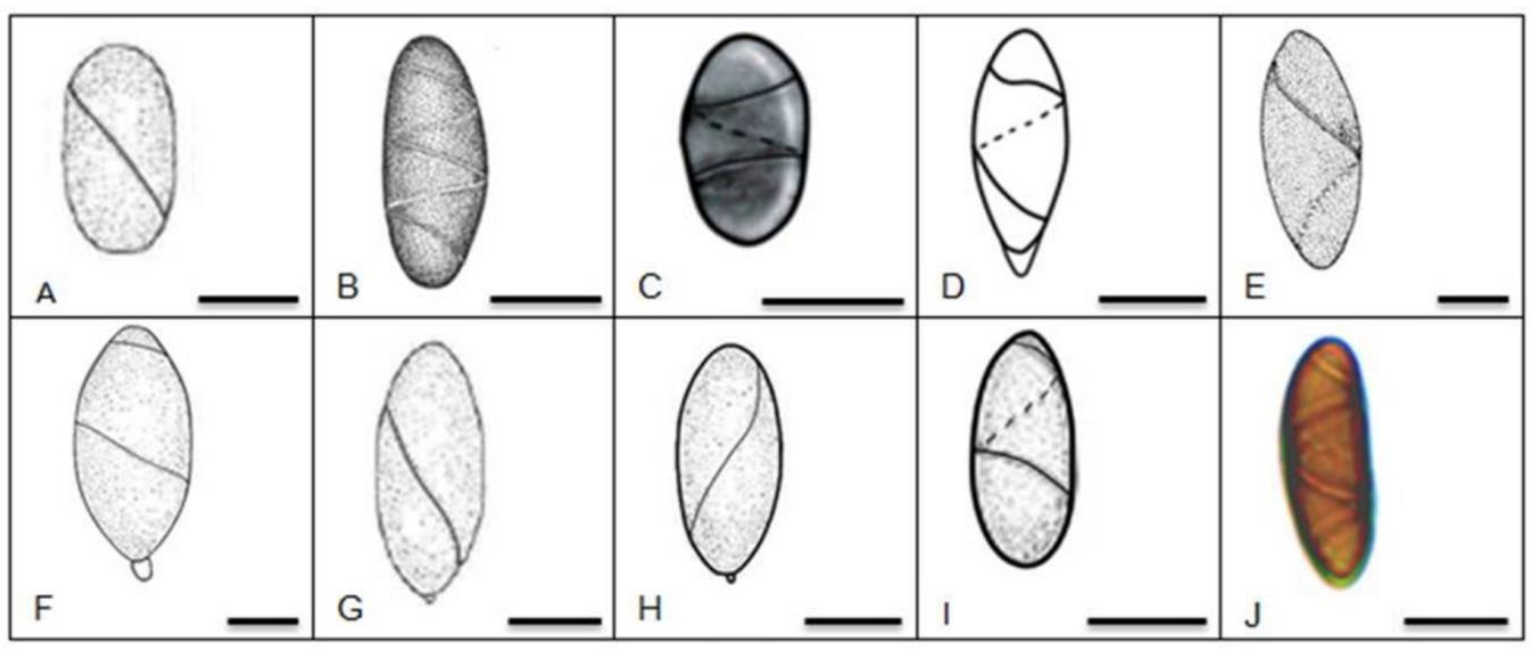

Fig. 2. Ascosporas de las especies de Helicogermslita redibujadas a partir de las fotos/ilustraciones de la bibliografía: A. H. aucklandica. (Petrini, 2003); B. H. celastri. (Hawksworth y Lodha, 1983); C. H. diversa. (Lee y Crous, 2003); D. H. fleischhakii. (Rappaz, 1995); E. H. gaudefroyi. (Laessoe y Spooner, 1993); F. H. gisbornia. (Petrini, 2003); G. H. johnstonii. (Petrini, 2003); H. H. mackenziei. (Petrini, 2003); I. H. valdiviensis. (Petrini, 1992);

J. Helicogermslita sp., espora observada en este trabajo. Barra $=10 \mu \mathrm{m}$ 
Tabla 1. Comparación entre la morfología de las ascosporas y la ecología de las especies aceptadas en el género Helicogermslita y las esporas halladas en la atmósfera de la ciudad de Bahía Blanca, Argentina.

\begin{tabular}{|c|c|c|c|c|c|c|}
\hline Especie & $\begin{array}{l}\text { Longitud } \\
(\mu \mathrm{m})\end{array}$ & $\begin{array}{c}\text { Diámetro } \\
(\mu \mathrm{m})\end{array}$ & Surcos & Apéndices & Distribución & Hospedante \\
\hline $\begin{array}{c}\text { H. aucklandica } \\
\text { (Rabenh) L. E. Petrini } \\
\text { Petrini (2003) }\end{array}$ & $19-23.5$ & $10-14$ & 1 & No & Nueva Zelanda & No consignado \\
\hline $\begin{array}{c}\text { H. celastri } \\
\text { (S. B. Kale \& S. V. S. } \\
\text { Kale) Lodha \& D. } \\
\text { Hawksw } \\
\text { Hawksworth \& Lodha } \\
\text { (1983) }\end{array}$ & $17-26$ & $8-10$ & $2-4$ & No & India & $\begin{array}{c}\text { Celastrus } \\
\text { paniculatus } \\
\text { Gymnosporia } \\
\text { montana } \\
\text { Lantana camara } \\
\text { Punica } \\
\text { granatum }\end{array}$ \\
\hline $\begin{array}{c}\text { H. diversa } \\
\text { S. Lee \& Crous } \\
\text { Lee \& Crous (2003) }\end{array}$ & $11-17.5$ & $6.5-11$ & $1-2$ & $\mathrm{Si}$ & Sudáfrica & $\begin{array}{l}\text { Leucadendron } \\
\text { sp. }\end{array}$ \\
\hline $\begin{array}{c}\text { H. fleischhakii } \\
\text { Laessoe \& Spooner } \\
\text { Laessoe \& Spooner } \\
\quad(1993)\end{array}$ & $\begin{array}{c}15.5- \\
17.5\end{array}$ & $9.5-10.5$ & 2 & $\mathrm{Si}$ & $\begin{array}{l}\text { Alemania } \\
\text { Francia }\end{array}$ & $\begin{array}{l}\text { Abies sp. } \\
\text { Pinus sp. }\end{array}$ \\
\hline $\begin{array}{c}H . \text { gaudefroyi } \\
\text { Laessoe \& Spooner } \\
\text { Laessoe \& Spooner } \\
(1993)\end{array}$ & $29-44$ & $12-17$ & 1 & No & Francia & Quercus sp. \\
\hline $\begin{array}{l}\text { H. gisbornia } \\
\text { L. E. Petrini } \\
\text { Petrini (2003) }\end{array}$ & $29.7-37.4$ & $14.4-18.2$ & $1 \frac{1 / 4}{4}$ & $\mathrm{Si}$ & Nueva Zelanda & $\begin{array}{l}\text { Beilschmiedia } \\
\text { tawa } \\
\text { Weinmannia } \\
\text { racemosa }\end{array}$ \\
\hline $\begin{array}{l}\text { H. johnstonii } \\
\text { L. E. Petrini } \\
\text { Petrini (2003) }\end{array}$ & $22.5-32$ & $9.5-14$ & 1 & $\mathrm{Si}$ & Nueva Zelanda & $\begin{array}{l}\text { Coprosma } \mathrm{sp} . \\
\text { Dracophyllum } \\
\text { longifolium }\end{array}$ \\
\hline $\begin{array}{c}\text { H. mackenziei } \\
\text { L. E. Petrini } \\
\text { Petrini (2003) }\end{array}$ & $20-25.5$ & $9.6-12.5$ & 1 & $\mathrm{Si}$ & Nueva Zelanda & Indeterminado \\
\hline $\begin{array}{c}\text { H. valdiviensis } \\
\text { Laessoe \& Spooner } \\
\text { Laessoe \& Spooner } \\
(1993)\end{array}$ & $17-21$ & $7.5-10$ & $11 / 2-2$ & No & Chile & Persea lingua \\
\hline $\begin{array}{l}\text { Helicogermslita } \\
\text { (Este trabajo) }\end{array}$ & $15-28.5$ & $6-12$ & $2-3$ & No & Argentina & $\begin{array}{c}\text { Desconocido. } \\
\text { Registrada en el } \\
\text { aire } \\
\end{array}$ \\
\hline
\end{tabular}

El índice anual de esporas correspondiente al tipo Helicogermslita fue de 145 esporas $/ \mathrm{m}^{3}$ (fig. 3), esto representó el $0.02 \%$ en relación al índice anual de esporas fúngicas (InAEs 592004 esporas $/ \mathrm{m}^{3}$ ). El registro de Helicogermslita fue continuo durante todo el año, con mayor representación en verano y en otoño, coincidente con los mayores valores de precipitación registrados (fig. 4). Los valores diarios registrados oscilaron entre 2 y 10 esporas $/ \mathrm{m}^{3}$ de aire (fig. 3). 


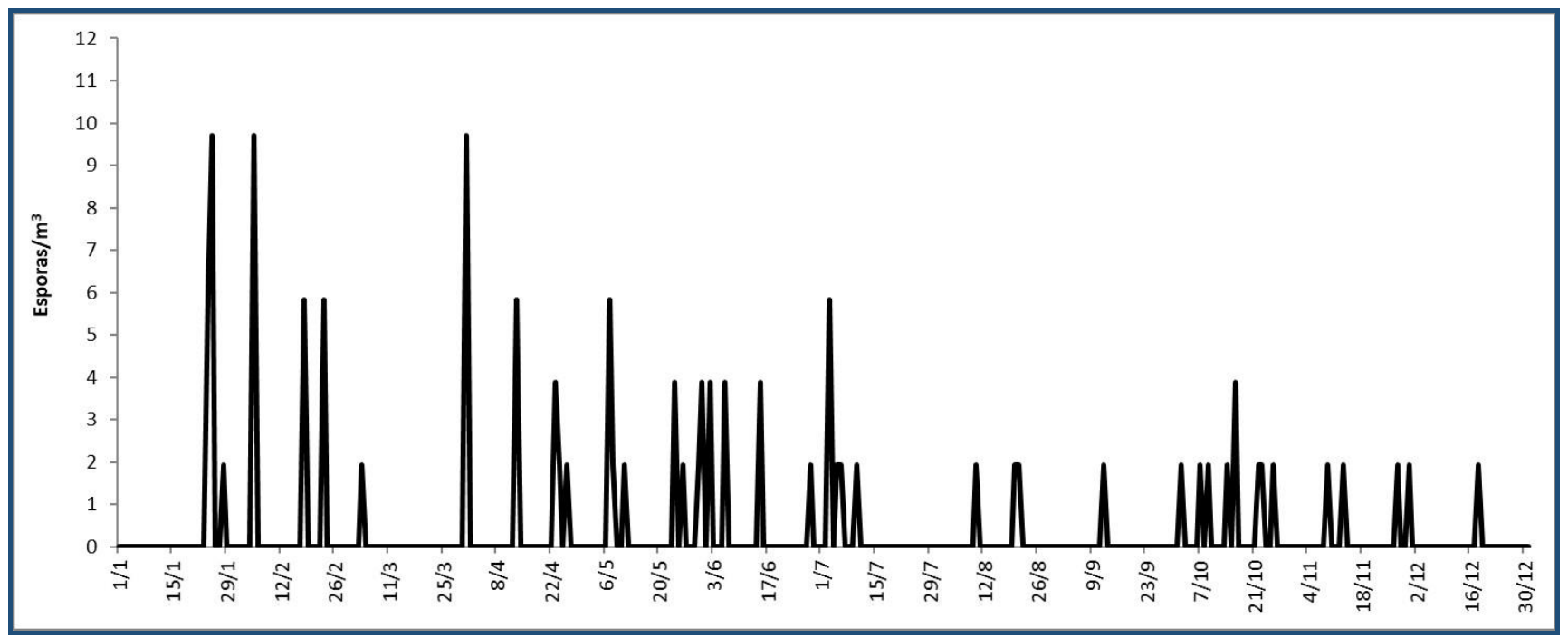

Fig. 3. Representación diaria del recuento de Helicogermslita (esporas $/ \mathrm{m}^{3}$ ) registradas durante el año de estudio en la atmósfera de la ciudad de Bahía Blanca, Argentina.

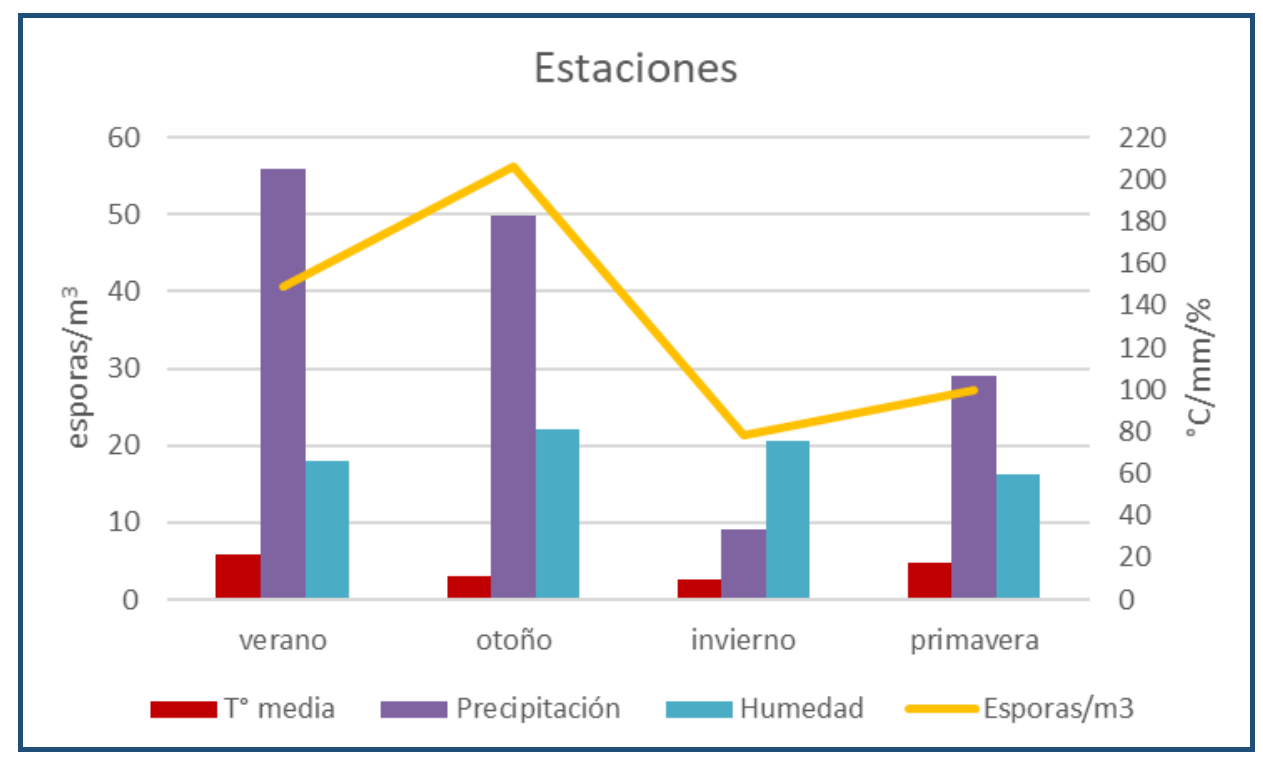

Fig. 4. Representación estacional del recuento de Helicogermslita (esporas $/ \mathrm{m}^{3}$ ) y variables meteorológicas [temperatura media $\left({ }^{\circ} \mathrm{C}\right)$, precipitación $(\mathrm{mm})$ y humedad relativa $(\%)$ ] en la atmósfera de la ciudad de Bahía Blanca, Argentina.

El test de normalidad realizado sobre los datos volumétricos diarios mostró que los datos no se ajustan a una distribución normal. Se decidió no realizar una transformación de los datos. Al analizar la relación entre la concentración diaria de esporas de Helicogermslita (esporas $/ \mathrm{m}^{3}$ ) y los parámetros meteorológicos, se registró una correlación altamente significativa con los valores diarios de humedad relativa $\left(0.26^{* *}\right)$ y una correlación significativa negativa con los valores diarios de temperatura máxima $(-0.12 *$; tabla 2$)$. El resto de los factores meteorológicos analizados (temperatura media, mínima, precipitación y velocidad de viento) no mostraron correlación significativa. 
Tabla 2. Correlación entre los factores meteorológicos y datos diarios de Helicogermslita (esporas $/ \mathrm{m}^{3}$ ).

Tm temperatura media, Tmax temperatura máxima, Tmin temperatura mínima, $\mathbf{P}$ precipitación, $\mathbf{H R}$ humedad relativa, VV velocidad de viento, $\mathbf{P - 1}$ precipitación del día anterior, $\mathbf{r}$ coeficiente de correlación, $\boldsymbol{n}$.s. no significativo.

\begin{tabular}{|l|c|c|}
\hline & N (días) & $\mathbf{r}$ \\
\hline Esporas $/ \mathrm{m}^{3} \& \mathrm{Tm}$ & 366 & $n . s$. \\
\hline Esporas $/ \mathrm{m}^{3} \& \mathrm{Tmax}$ & 366 & $-0.12^{*}$ \\
\hline Esporas $/ \mathrm{m}^{3} \& \mathrm{Tmin}$ & 366 & $n . s$. \\
\hline Esporas $/ \mathrm{m}^{3} \& \mathrm{P}$ & 85 & $n . s$. \\
\hline Esporas $/ \mathrm{m}^{3} \& \mathrm{HR}$ & 366 & $0,26^{* *}$ \\
\hline Esporas $/ \mathrm{m}^{3} \& \mathrm{VV}$ & 366 & $n . s$. \\
\hline Esporas $/ \mathrm{m}^{3} \& \mathrm{P}-1$ & 84 & $n . s$. \\
\hline
\end{tabular}

\section{DISCUSIÓN}

Helicogermslita es uno de los géneros menos estudiados entre los denominados xilariales microscópicos (Daranagama et al., 2016). Varias especies son solo conocidas de la colección tipo y raramente han sido reencontradas. Sin embargo, el surco germinativo helicoidal que poseen sus ascosporas, facilita su reconocimiento, aún en sustratos o ambientes diferentes a aquellos en los cuales las especies fueron originalmente descriptas. Es así que la presencia de Helicogermslita ha sido registrada en sedimentos (van-Geel et al., 2011), turba (Prager et al., 2012), mieles (Magyar et al., 2016) y en muestras de aire (Grant-Smith, 1990). En este último medio, parecen ser poco frecuentes. Sin embargo, en España han sido mencionadas en varios estudios aerobiológicos (Díez-Herrero et al., 2006; Nieto-Lugilde, 2008; Docampo et al., 2011; Elvira-Rendueles et al., 2013), pero sin intentar asignarles identidad o afinidad específicas. En Argentina, el hallazgo de ascosporas de Helicogermslita en muestras de aire de la ciudad de Bahía Blanca constituyó la primera cita del género para el país y la segunda para el cono Sur (Castillo et al., 2014).

Para la identificación de especies en Helicogermslita, se utilizan, entre otros caracteres, atributos morfológicos de las ascosporas, tales como su tamaño, forma, presencia de apéndices y vainas, y características del surco (Hawksworth y Lodha, 1983; Laessoe y Spooner, 1993; Lee y Crous, 2003; Petrini, 1992, 2003). De acuerdo a ellos, las esporas halladas en el aire de Bahía Blanca mostraron gran similitud con las de $H$. celastri. Solo H. valdiviensis había sido citada anteriormente en Sudamérica, pero sus ascosporas son más pequeñas y el surco presenta menos giros. La identidad de las ascosporas estudiadas en este trabajo se mantiene, sin embargo, con asignación abierta (Helicogermslita cf. celastri), ya que la imposibilidad de estudiar las restantes estructuras reproductivas en detalle impide una identificación más precisa.

Varios estudios aerobiológicos han demostrado que los restos fúngicos que se registran en un ambiente son, en la mayoría de los casos, estrictamente de ocurrencia local (van Geel y Aptroot, 2006; Shumilovskikh et al., 2017;). Las esporas se depositan en general donde se originaron o a corta distancia del sitio de esporulación (Medeanic y Silva, 2010), ya que muchas especies de hongos tienen restringida su capacidad para ser transportadas por el aire, por lo que mantienen una buena correlación ambiental como biota local con el sitio depositacional (Medeanic et al., 2004). Por ello, es esperable que los cuerpos reproductivos que originaron las ascosporas encontradas en el aire en este trabajo se encuentren en algunas de las plantas que integran la flora cercana al sitio de ubicación del equipo de monitoreo. Varios de los sustratos sobre los que 
ha sido registrada $H$. celastri son abundantes en la localidad en estudio (Bahía Blanca) donde han sido introducidos como ornamentales, por ejemplo, Lantana y algunas especies de Pinus. Si bien estas especies arbóreas son también comunes en la flora de otras ciudades del país, la presencia de Helicogermslita no ha sido mencionada en los escasos estudios aerobiológicos previos que consignan en forma detallada las esporas de origen fúngico (Mallo et al., 2011; Nuñez-Otaño et al., 2015).

La baja representación de Helicogermslita cf. celastri en relación al resto de las esporas detectadas en la atmósfera, es consistente con lo observado en otros estudios, en los cuales su representación también fue muy baja (0.01\%) (Díez-Herrero et al., 2006; Docampo et al., 2011). Siguiendo las clases determinadas por Martínez-Blanco et al. (2016), la espora en estudio representa el $0.48 \%$ de los tipos esporales incluidos en la clase "baja abundancia", con InAES 145 esporas $/ \mathrm{m}^{3}$ frente a tipos más abundantes como Alternaria $($ InAEs $=42707$ esporas $/ \mathrm{m}^{3}$ ) y Cladosporium $\left(\mathrm{InAEs}=385556\right.$ esporas $\left./ \mathrm{m}^{3}\right)$.

La variación de las ascosporas en el aire exterior es influenciada por la presencia de las estructuras reproductivas (fuente de aporte de esporas) y por los factores meteorológicos que contribuyen a la dispersión de partículas en la atmósfera (humedad, viento, temperatura, etc.).

Las ascosporas son generalmente liberadas por un mecanismo activo, asociado con la lluvia y la humedad, cuando la presión osmótica en el interior del asco aumenta (Rivera-Mariani y Bolaños-Rosero, 2012). Almaguer et al. (2014), Haskouri et al. (2016) y Ferro et al. (2018), encontraron que las precipitaciones y la humedad relativa fueron las variables más influyentes en la aparición de esporas fúngicas en la atmósfera. Esto es consistente con los hallazgos del presente estudio en lo que respecta a la relación de los factores meteorológicos y el contenido de esporas.

La correlación positiva con la humedad relativa y la correlación negativa con la temperatura máxima observadas en este estudio son consistentes con lo observado por Hernández-Trejo et al. (2012) al estudiar la relación entre las variables y la concentración de ascosporas en el aire de una ciudad del sudoeste de España.

El presente trabajo de investigación constituye un aporte que complementa los escasos estudios realizados en Argentina sobre la dispersión de ascosporas en el aire. Como señalaron Hjelmroos (1993), De-Antoni et al. (2006) y Grinn-Gofroń y Bosiacka (2015), resulta necesario efectuar más investigaciones para establecer parámetros generales que permitan dilucidar con mayor claridad los fenómenos de dispersión de las ascosporas en la atmósfera. En base a lo observado en este estudio, se propone continuar con un monitoreo a largo plazo para determinar la relación con las variables meteorológicas que, ante la escasez de los datos, no han mostrado un resultado significativo desde el punto de vista estadístico.

\section{CONCLUSIONES}

El tipo esporal Helicogermslita hallado en el aire de la ciudad de Bahía Blanca fue identificado como Helicogermslita cf. celastri. Esta ascospora se encontró presente en mayor abundancia durante los meses de verano y otoño. Su dispersión fue influenciada por la precipitación y la humedad, factores meteorológicos que favorecen la liberación de las esporas al aire. 


\section{AGRADECIMIENTOS}

Este estudio fue financiado por el Consejo Nacional de Investigaciones Científicas y Técnicas (CONICET, Argentina) y la Universidad Nacional del Sur. Los autores agradecen la colaboración de la doctora Melina Calfuán y Santino Frapiccini en la edición de las imágenes.

\section{LITERATURA CITADA}

Almaguer, M., Aira, M., Rodríguez-Rajo, F. y Rojas, T. (2014). Temporal dynamics of airborne fungi in Havana (Cuba) during dry and rainy seasons: influence of meteorological parameters. International Journal of Biometeorology, 58(7), 1459-1470. https://doi.org/10.1007/s00484-013-0748-6

Castillo, L. A., Murray, M. G. y Bianchinotti, M. V. (2014). Aeróspora fúngica: Diversidad y abundancia en el aire de la ciudad de Bahía Blanca durante marzo de 2013. XIII Congreso Argentino de Micología y 1ra Reunión de la Asociación Micológica Carlos Spegazzini. Buenos Aires, Argentina.

Daranagama, D. A., Camporesi, E., Jeewon, R., Liu, X., Stadler, M., Lumyong, S. y Hyde, K. D. (2016). Taxonomic Rearrangement of Anthostomella (Xylariaceae) Based on a Multigene Phylogeny and Morphology. Cryptogamie Mycologie, 37(4), 509-538.

De-Antoni, Z. B., Valencia-Barrera, R., Vergamini-Duso, S. y Fernández-González, D. (2006). Fungal spores prevalent in the aerosol of the city of Caxias do Sul, Rio Grande do Sul, Brazil, over a 2-year period (2001-2002). Aerobiologia, 22(2), 119-126. https://doi.org/10.1007/s10453-006-9022-2

Díez-Herrero, A., Sabariego-Ruiz, S., Gutiérrez-Bustillo, M. y Cervigón-Morales, P. (2006). Study of airborne fungal spores in Madrid, Spain. Aerobiologia, 22(2), 135-142. https://doi.org/10.1007/s10453-006-9025-z

Docampo, S., Trigo, M., Recio, M., Melgar, M., García-Sánchez, J. y Cabezudo, B. (2011). Fungal spore content of the atmosphere of the Cave of Nerja (southern Spain): Diversity and origin. Science of the Total Environment, 409(4), 835-843. https://doi.org/10.1016/j.scitotenv.2010.10.048

Elvira-Rendueles, B., Moreno, J., Garcia-Sanchez, A., Vergara, N., Martinez-Garcia, M. J. y Moreno-Grau, S. (2013). Air-spore in Cartagena, Spain: Viable and non-viable sampling methods. Annals of Agricultural and Environmental Medicine, 20(4), 664-671.

Ferro, R., Nunes, C., Caeiro, E., Camacho, I., Paiva, M. y Morais-Almeida, M. (2018). Aeromicologia de Lisboa e a sua relação com os fatores meteorológicos. Revista Portuguesa de Imunoalergologia, 26, 21-33.

Galán-Soldevilla, C., Alcázar-Teno, P., Cariñanos-González, P. y Domínguez-Vilches, E. (2007). Manual de calidad y gestion de la red española de aerobiologia. Universidad de Córdoba, España.

Galán, C., Ariatti, A., Bonini, M., Clot, B., Crouzy, B., Dahl, A., Fernandez-González, D., Frenguelli, G., Gehrig, R., Isard, S., Levetin, E., Li, D. W., Mandrioli, P., Rogers, C. A., Thibaudon, M., Sauliene, I. y Skjoth, C., Smith, M. y Sofiev, M. (2017). Recommended terminology for aerobiological studies. Aerobiologia, 33(3), 293-295. https://doi.org/10.1007/s10453-017-9496-0

Grant-Smith, E. (1990). Sampling and Identifying allergenic pollens and molds. San Antonio, Texas: Blewstone Press.

Grinn-Gofroń, A. y Bosiacka, B. (2015). Effects of meteorological factors on the composition of selected fungal spores in the air. Aerobiologia, 31(1), 63-72. https://doi.org/10.1007/s10453-014-9347-1

Haskouri, F., Bouziane, H., Trigo, M., Kadiri, M. y Kazzaz, M. (2016). Airborne ascospores in Tetouan (NW Morocco) and meteorological parameters. Aerobiologia, 32(4), 669681. https://doi.org/10.1007/s10453-016-9440-8 
Hawksworth, D. L. y Lodha, B. C. (1983). Helicogermslita, a new stromatic xylariaceous genus with a spiral germ slit from India. Transactions of the British Mycological Society, 81(1), 91-96. https://doi.org/10.1016/S0007-1536(83)80208-3

Hernández Trejo, F., Muñoz Rodríguez, A. F., Tormo Molina, R. y Silva Palacios, I. (2012). Airborne ascospores in Mérida (SW Spain) and the effect of rain and other meteorological parameters on their concentration. Aerobiologia, 28(1), 13-26. https://doi.org/10.1007/s10453-011-9207-1

Hjelmroos, M. (1993). Relationship between airborne fungal spore presence and weather variables: Cladosporium and alternarla. Grana, 32(1), 40-47. https://doi.org/10.1080/00173139309436418

Kottek, M., Grieser, J., Beck, C., Rudolf, B. y Rubel, F. (2006). World Map of the KöppenGeiger climate classification updated. Meteorologische Zeitschrift, 15(3), 259-263. https://doi.org/10.1127/0941-2948/2006/0130

Laessoe, T. y Spooner, B. M. (1993). Rosellinia \& Astrocystis ( Xylariaceae ): new species and generic concepts. Kew Bulletin, 49(1), 1-70.

Lee, S. y Crous, P. W. (2003). A new species of Helicogermslita from South Africa.pdf. Sydowia, 55(1), 109-114.

Magyar, D., Mura-Mészáros, A. y Grillenzoni, F. (2016). Fungal diversity in floral and honeydew honeys. Acta Botanica Hungarica, 58(1-2), 145-166. https://doi.org/10.1556/034.58.2016.1

Mallo, A. C., Nitiu, D. S. y Gardella-Sambeth, M. C. (2011). Airborne fungal spore content in the atmosphere of the city of la Plata, Argentina. Aerobiologia, 27(1), 77-84. https://doi.org/10.1007/s10453-010-9172-0

Martínez-Blanco, X., Tejera, L. y Beri, Á. (2016). First volumetric record of fungal spores in the atmosphere of Montevideo City, Uruguay: a 2-year survey. Aerobiologia, 32(2), 317-333. https://doi.org/10.1007/s10453-015-9403-5

Medeanic, S., García, M. J. y Steveaux, J. C. (2004). The importance of fungal and algal palynomorphs for paleoenvironment reconstructions using sediments obtained in the upper Parana river. Geociencias, 9(6), 19-37.

Medeanic, S. y Silva, M. B. (2010). Indicative value of nonpollen palynomorphs (NPPs) and palynofacies for palaeoreconstructions. International Journal of Coal Geology, 84, 248-257.

Nieto-Lugilde, D. (2008). Estudio aerobiológico de la zona costera de la provincia de Granada (Motril): evolución de las concentraciones de polen y esporas. Universidad de Granada, España.

Nuñez-Otaño, N., di-Pasquo, M. y Muñoz, N. (2015). Airborne fungal richness: proxies for floral composition and local climate in three sites at the El Palmar National Park (Colón, Entre Ríos, Argentina). Aerobiologia, 31(4), 537-547. https://doi.org/10.1007/s10453-015-9382-6

Petrini, L. E., Petrini, O. y Fisher, P. J. (1987). Anthostomella calligoni, an endophyte of Suaeda fruticosa in Dorset. Transactions of the British Mycological Society, 89(3), 387-389. https://doi.org/10.1016/s0007-1536(87)80124-9

Petrini, L. E. (1992). Rosellinia species of the temperate zones. Sydowia, 44(2), 169-281.

Petrini, L. E. (2003). Rosellinia and related genera in New Zealand. New Zealand Journal of Botany, 41, 71-138. https://doi.org/10.1080/0028825X.2003.9512833

Prager, A., Theuerkauf, M., Couwenberg, J., Barthelmes, A., Aptroot, A. y Joosten, H. (2012). Pollen and non-pollen palynomorphs as tools for identifying alder carr deposits: A surface sample study from NE-Germany. Review of Palaeobotany and Palynology, 186, 38-57. https://doi.org/10.1016/j.revpalbo.2012.07.006

Rappaz, F. (1995). Anthostomella and related Xylariaceous fungi on hard wood from Europe and North America. Mycologia Helvetica, 7, 99-168.

Rivera-Mariani, F. E. y Bolaños-Rosero, B. (2012). Allergenicity of airborne basidiospores and ascospores: need for further studies. Aerobiologia, 28(2), 83-97. https://doi.org/10.1007/s10453-011-9234-y 


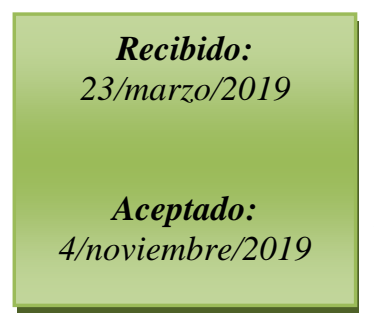

Shumilovskikh, L., Ferrer, A. y Schlütz, F. (2017). Non-pollen palynomorphs notes: 2. Holocene record of Megalohypha aqua-dulces, its relation to the fossil form genus Fusiformisporites and association with lignicolous freshwater fungi. Review of Palaeobotany and Palynology, 246, 167-176. https://doi.org/10.1016/j.revpalbo.2017.07.002

van-Geel, B., Gelorini, V., Lyaruu, A., Aptroot, A., Rucina, S., Marchant, R., SinningheDamsté, J. S. y Verschuren, D. (2011). Diversity and ecology of tropical African fungal spores from a 25,000-year palaeoenvironmental record in southeastern Kenya. Review of Palaeobotany and Palynology, 164(3-4), 174-190. https://doi.org/10.1016/j.revpalbo.2011.01.002

van-Geel, B. y Aptroot, A. (2006). Fossil ascomycetes in Quaternary deposits. Nova Hedwigia, 82(3-4), 313-329. https://doi.org/10.1127/0029-5035/2006/0082-0313

Whalley, A. J. S. (1996). The xylariaceous way of life. Mycological Research, 100(8), 897922. https://doi.org/10.1016/S0953-7562(96)80042-6 